\title{
Stratégie d'écrêtement des crues en Loire moyenne Méthode, limites et impacts
}

\author{
A. Bachoc \\ Service de Bassin Loire Bretagne / Direction régionale de l'environnement du Centre
}

\section{R. Thépot}

Etablissement Public d'Aménagement de la Loire et de ses Affluents (EPALA)

\section{N-G Camp'huis}

Equipe pluridisciplinaire du plan Loire grandeur nature

(Agence de l'eau Loire Bretagne, EPALA, Etat)

\section{1 UNE SITUATION ET UNE EXPÉ- RIENCE HÉRITÉES DU PASSÉ}

1.1 Levées, vals et déversoirs

Des levées battues en brèches par les crues

Depuis l'apparition des premières turcies puis des levées, la politique de gestion de la Loire alterne :

- les phases de rehaussement des levées (en 1663, jusqu'à 3 toises ou $6 \mathrm{~m}$ sous ordre de Colbert; en 1708 jusqu'à 22 pieds ou $7.10 \mathrm{~m}$ ) dans l'espoir de toujours contenir les crues dans le lit endigué,

- et les moments où l'homme, rappelé à la dure réalité par des crues exceptionnelles, a tenté d'instaurer dans les levées, des déchargeoirs ou des déversoirs pour ménager " la part de l'eau " dans les vals (Louis XIII, Louis XV, 1867).

Le système de levées s'est mis en place progressivement et par à-coups, autant pour protéger des espaces agricoles riches ou des villes fondées sur le commerce fluvial, que pour fixer le cours de la Loire le long de certains ports (Fourchambault, la Charité, Briare, Gien, Orléans, etc.). Aujourd'hui sur les $450 \mathrm{~km}$ de Loire moyenne (Bec d'Allier - Bec de Maine), $700 \mathrm{~km}$ de levées encadrent le fleuve. Aucune n'a de fondation ; les plus sensibles se composent de plusieurs levées emboîtées comme des poupées russes, héritage des différentes tentatives de rehaussement (fig. 1).

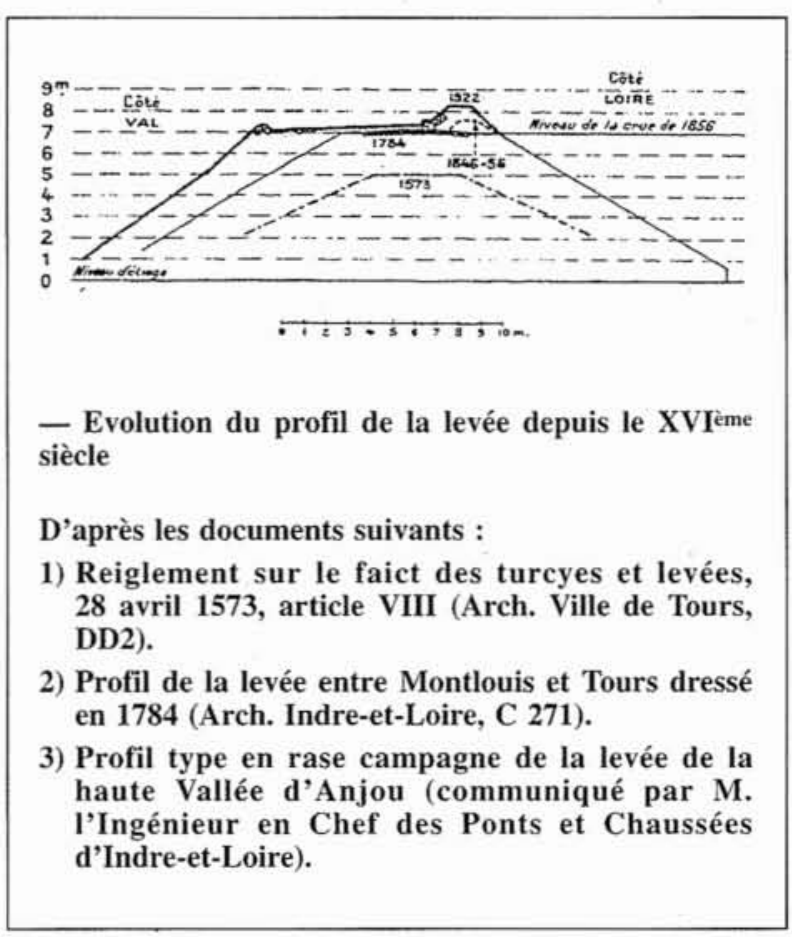

1. Coupe d'une levée (source: Histoire des levées, de Dion) 
Le système est fragile, puisque la crue de 1846 a ouvert 100 brèches entre Briare et Langeais et que celle de 1856 en a provoqué 160 , totalisant $23 \mathrm{~km}$ de levées détruits et n'épargnant aucun val. Une expertise de Z. Gasowski révèle que le tiers seulement des ruptures trouve son origine dans la surverse et qu'un autre tiers a eu lieu alors que l'eau était restée à plus de $10 \mathrm{~cm}$ sous la crête de la levée (on doit alors penser aux renards).

Pourtant le système doit résister, d'abord pour éviter l'inondation des zones de plus en plus vulnérables qu'il protège aujourd'hui. Dans les vals munis de déversoir, il doit aussi résister pour conserver la capacité d'écrêtement au val, en évitant qu'une rupture intempestive ne le noie avant le moment optimal de fonctionnement du déversoir de sécurité.

\section{Donc des vals}

Les levées délimitent près de 100.000 ha de vals en Loire moyenne, présentant une grande diversité. Certains sont totalement clos et peuvent paraître insubmersibles, comme celui de Givry, face à Fourchambault, ceux de Tours ou d'Authion (70 km continus de levées pour ce dernier) ; les levées n'y seraient pas submergées avant 6 ou $6500 \mathrm{~m}^{3} / \mathrm{s}$. D'autres sont ouverts à l'aval, du fait d'une confluence avec un petit affluent drainant le val ; les crues y pénètrent par remous, pour des niveaux de crue variables (Léré, $S^{t}$ Benoît, Orléans, Avaray, Cisse (Amboise), Vieux Cher): $: \AA$ Gien et Blois, des déchargeoirs inondent le val limitrophe dès $3500 \mathrm{~m}^{3} / \mathrm{s}$. D'autres vals sont munis de déversoirs (fig. 2).

\section{Des déversoirs plutôt que des brèches}

Suite aux crues de 1519, 1527, 1549, 1570, 1581, 1608 et 1628, le Conseil d'Etat du Roi marque une pause dans le relèvement systématique des levées pour se protéger des crues. Il prévoit le 19 décembre 1629 , la réalisation de six déchargeoirs comparables à celui mis en chantier à Blois vers 1602. Mais les bourgeois (au sens « habitants des bourgs ", principalement ceux d'Orléans, Blois, Amboise et Tours) qui ont des intérêts dans les vignes ou les cultures maraîchères protégées par les levées, s'opposent si bien au programme qu'aucun des déchargeoirs n'est finalement réalisé.

La cruelle série de $1707,1709,1710$ et 1711 incite à nouveau à réaliser, dès 1711 , ce programme de déchargeoirs. Mais il se révèle insuffisamment efficace lors de la crue de 1733 et tous sont refermés sauf ceux de Gien et Blois, qui fonctionnent aujourd'hui dès $3500 \mathrm{~m}^{3} / \mathrm{s}$ et protègent ainsi les ponts.

Suite aux trois crues du XIX ${ }^{\text {ème }}$ siècle, l'ingénieur Comoy propose de réaliser des déversoirs avec seuils fusibles, idée très judicieuse pour provoquer un écrêtement très rapide au passage du maximum de la crue (fig. 3). La modélisation de 1982 a montré combien Comoy avait calé les plates-formes et les crêtes des fusibles de chaque déversoir à une cote pertinente, opération d'autant plus difficile que les ruptures généralisées des levées ne lui permettaient pas de disposer des cotes de déversement correspondant au passage d'une crue entre des levées intactes.

Il prévoit 20 déversoirs, ouvrant aux crues 18 vals et 44000 ha, dans des sites réputés pour leurs brèches à chaque crue exceptionnelle. Sept seulement sont réalisés entre 1870 et 1891 (fig. 2). Les oppositions locales ont raison des autres déversoirs ; des vals systématiquement inondés par rupture de levée, comme ceux d'Authion ou de Cisse, ne sont finalement pas équipés, laissant entier le danger d'une brèche inopinée et donc beaucoup plus dévastatrice.

\subsection{Un fleuve modelé pour la navigation et par les extractions}

Les levées existent en partie pour canaliser la Loire et favoriser la navigation. Ponctuellement des ouvrages particuliers chenalisent davantage le fleuve, au passage des grandes agglomérations et aux abords des ports ou des jonctions Loire - canal latéral. Ces ouvrages (chevrettes, duits, épis, etc.) se retrouvent à Fourchambault, la Charité, Ousson, Châtillon, Orléans, Tours, etc.

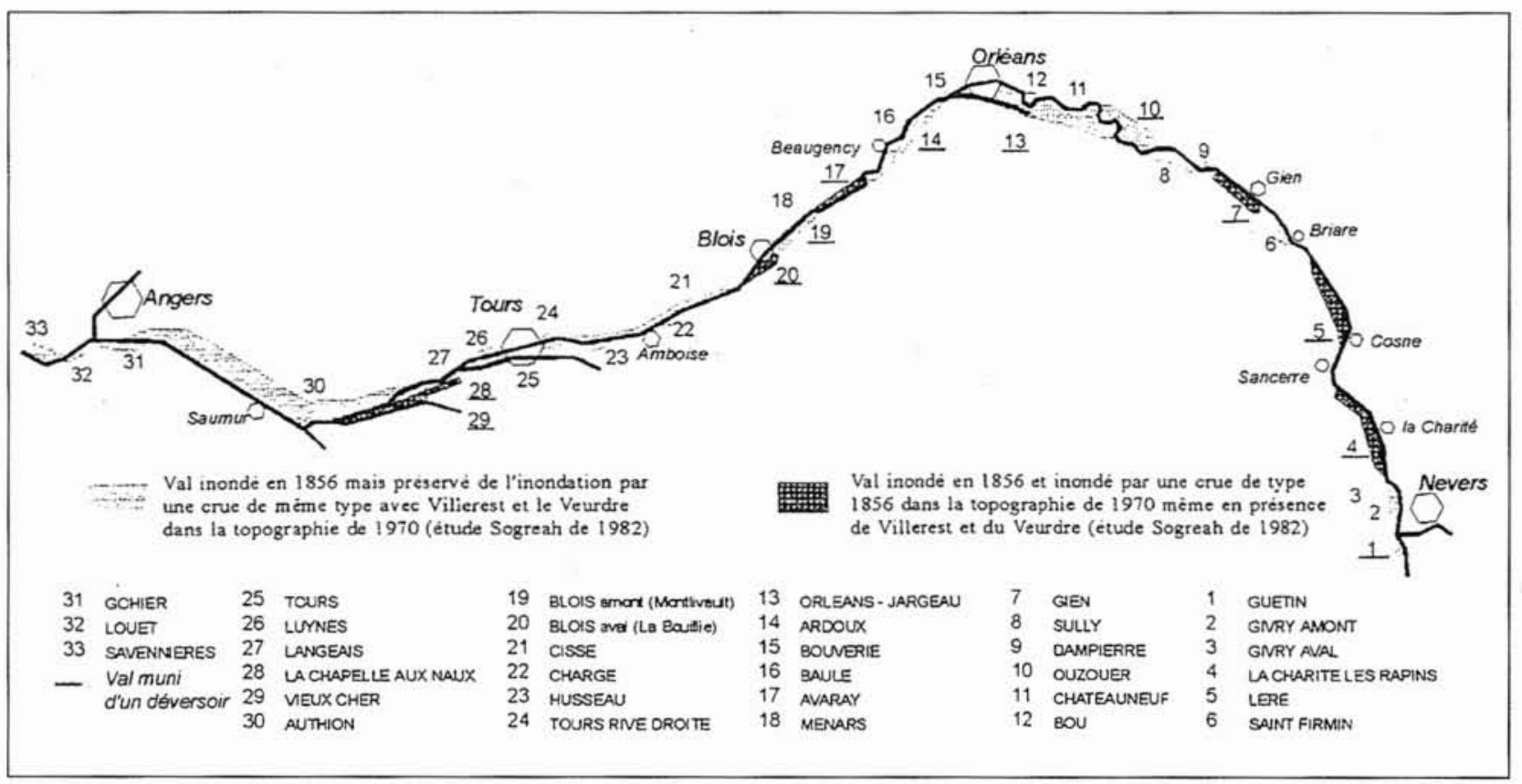

2. Carte des vals et des déversoirs en Loire moyenne 


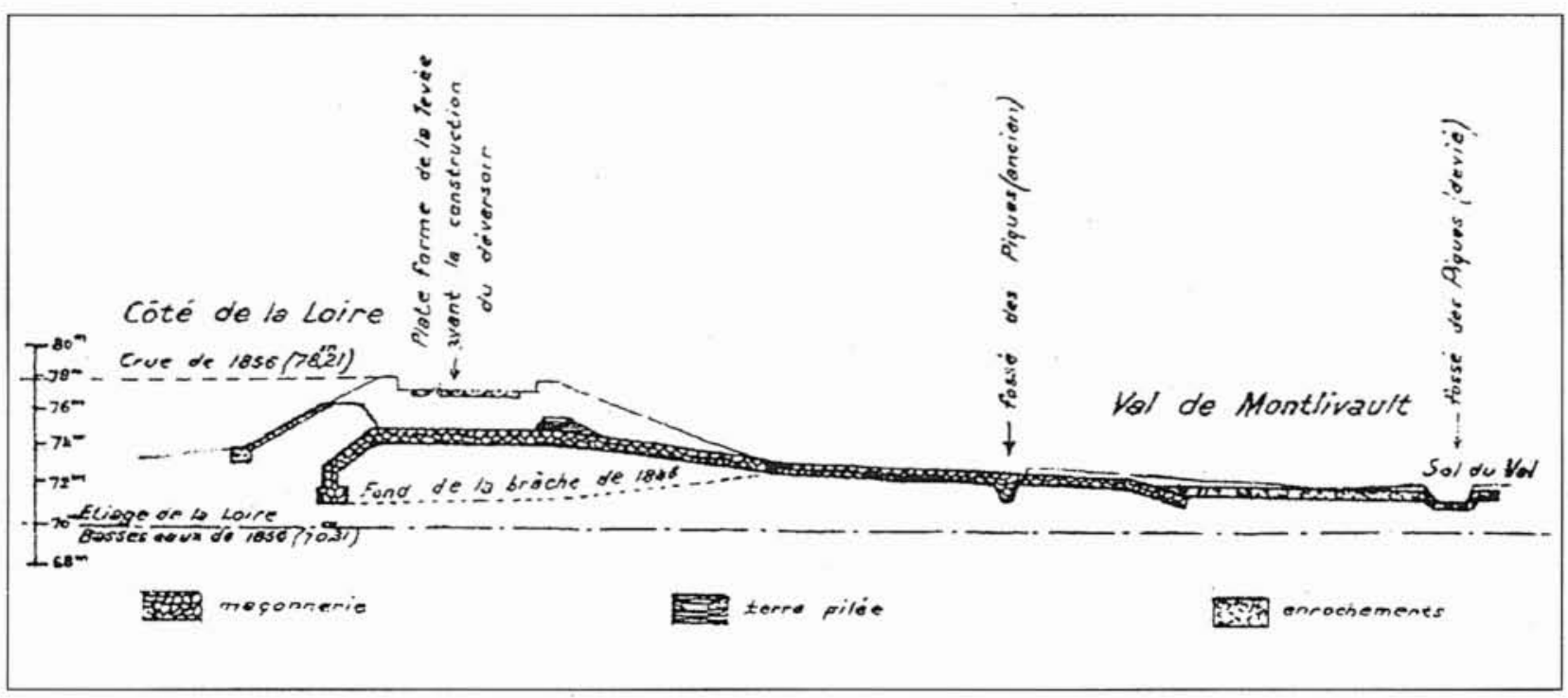

3. Coupe transversale du déversoir avec seuil fusible de Montlivault (Source : Dion)

Ils ont induit localement une chenalisation et un approfondissement de la Loire, qui se traduit par un abaissement de la ligne d'eau à l'étiage, qui se répercute vers l'amont. Les extractions massives de matériaux autour des principales agglomérations (200 millions de tonnes en 40 ans pour un apport moyen annuel de 0,5 million de tonne) ont considérablement accéléré le processus engagé et l'abaissement de la nappe.

Le lit endigué se trouve moins sollicité par les crues de faible période de retour. La végétation croît à l'abri de ces crues et provoque le dépôt des sédiments qu'elles apportent : le lit s'exhausse peu à peu et se couvre progressivement d'une végétation herborée, puis arbustive et ligneuse, qui le rend de plus en plus "rugueux » vis-à-vis des écoulements, particulièrement lors des crues. Moins fréquemment inondée, fondée sur une nappe phréatique qui tend à baisser du fait de l'enfoncement de la ligne d'eau, la végétation perd de sa richesse écologique et se banalise. Les bras morts s'assèchent et se ferment. L'ensemble de l'écosystème évolue vers un appauvrissement (fig. 4).

Les ouvrages de navigation, éléments importants du patrimoine et vestiges d'une ère d'aménagement fluvial, nécessitent une entretien particulier et parfois des modifications de fonctionnement hydraulique. On évite ainsi qu'ils freinent les crues, après avoir favorisé le développement d'une végétation qui peut, par ailleurs, constituer un paysage apprécié, particulièrement dans les villes. A Fourchambault - Cours les Barres, la Charité et Ousson - Châtillon, la DIREN a engagé des études (avec modélisation hydraulique bidimensionnelle) pour préciser les travaux de modifications pertinents et leur impact à moyen terme sur la propagation des crues, la ligne d'eau à l'étiage et les milieux naturels influencés.

\subsection{Des ouvrages écrêteurs sur le haut bassin}

L'intérêt d'un écrêteur des crues à leur origine dans le haut du bassin versant de la Loire ou de l'Allier est reconnu depuis longtemps. Les crues de 1707 à 1711 l'ont mis en lumière puisqu'on a attribué partiellement leur importance à l'arasement, pour faciliter la navigation, de deux seuils rocheux à Pinay et la Roche, en amont immédiat de Villerest. Deux barrages reconstruits sur ces sites au $\mathrm{XVIII}^{\mathrm{e} m \mathrm{me}}$ siècle ont eu un impact certain sur les crues du $\mathrm{XIX}^{\mathrm{ème}}$. En juillet 1847, l'ingénieur en chef Boulangé estime le volume retenu à 108 millions de $\mathrm{m}^{3}$, avec abaissement du débit maximum de près de $2000 \mathrm{~m}^{3} / \mathrm{s}$. Il relance l'idée de

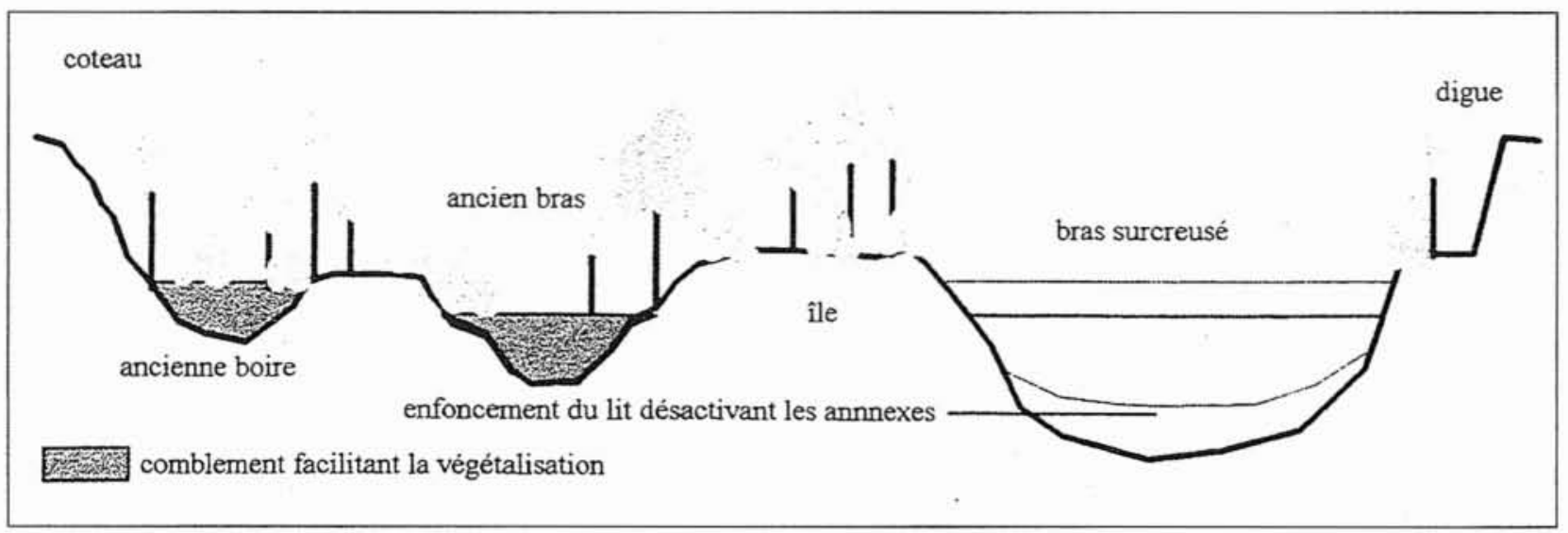

4. Comblement et végétalisation des annexes après enfoncement du lit 
réaliser 24 retenues d'un coût unitaire de 100000 francs sur 12 affluents du haut bassin.

Comoy approfondit la question et recense en 1867, 85 sites de barrages pouvant emmagasiner 520 millions de $\mathrm{m}^{3}$, pour un coût de 100 millions de francs de l'époque (soit le triple du coût de son programme de 20 déversoirs). Aucun ouvrage ne sera réalisé avant celui de Villerest en 1984.

\subsection{Dans les vals, des enjeux réglementés mais consi- dérables}

\section{Des enjeux qui ont explosé en 30 ans}

Quelques chiffres suffisent pour montrer comment les enjeux ont explosé depuis 1965 :

- en 1856, 26000 sinistrés et 57 millions de francs de dégâts (1,1 milliard actuel).

- en 1995,250000 personnes à évacuer et de 7 à 24 milliards de francs de dégâts (dépendant de l'ampleur de l'inondation, du niveau de rupture des levées ainsi que des atteintes aux biens privés, aux infrastructures économiques et publiques et aux réseaux).

\section{Les démarches de contrôle de l'urbanisation en zone inondable}

Une loi de 1934 définissant des plans de surfaces submersibles s'est appliquée par décret publié entre 1958 et 1974 suivant les départements. Ce seul retard traduit bien les difficultés à réglementer l'occupation du sol dans les zones inondables. Quelques années après, pouvoirs publics et élus locaux, oubliant le risque que cette loi cherchait à rappeler, laissaient construire en zone inondable. A une ambition justifiée de diminuer les enjeux exposés aux inondations certaines, répondait l'échec de la résistance à l'attrait des zones inondables.

L'application, par l'Etat, de la loi de 1987 sur l'information des populations sur les risques naturels majeurs se traduit aujourd'hui par la publication des atlas des zones inondables entre le Bec d'Allier et Nantes. La procédure de projet de protection qualifié de projet d'intérêt général par les préfets, se met en place progressivement et amène les communes à modifier leur plan d'occupation des sols pour ne plus construire dans les « champs d'expansion des crues à préserver de toute urbanisation naturelle " et pour contenir les enjeux exposés dans les zones déjà urbanisées.

\section{II 目 COMMENT GÉRER LES CRUES EN LOIRE MOYENNE AUJOURD'HUI ?}

\subsection{L'ampleur du phénomène}

En 1856, la crue a atteint $7600 \mathrm{~m}^{3} / \mathrm{s}$ au Bec d'Allier; elle a dépassé $1000 \mathrm{~m}^{3} / \mathrm{s}$ pendant 10 jours pour 1,6 milliard de $\mathrm{m}^{3}$ écoulés (fig. 5).

Ramener une telle crue à un niveau maximum de $6000 \mathrm{~m}^{3} / \mathrm{s}$ oblige à différer l'écoulement de plus de 100 millions de $\mathrm{m}^{3}$; la ramener à $5000 \mathrm{~m}^{3} / \mathrm{s}$ conduit à différer près de 250 millions de $\mathrm{m}^{3}$.

\subsection{Le lit endigué}

\section{Tenir compte de l'enfoncement}

Une modélisation de la crue de 1856 sur la Loire moyenne, dans une topographie datant de 1965 à 1980, entreprise en 1982, a révélé que l'enfoncement du lit dépassant (en moyenne transversale) le mètre à certains endroits, modifie

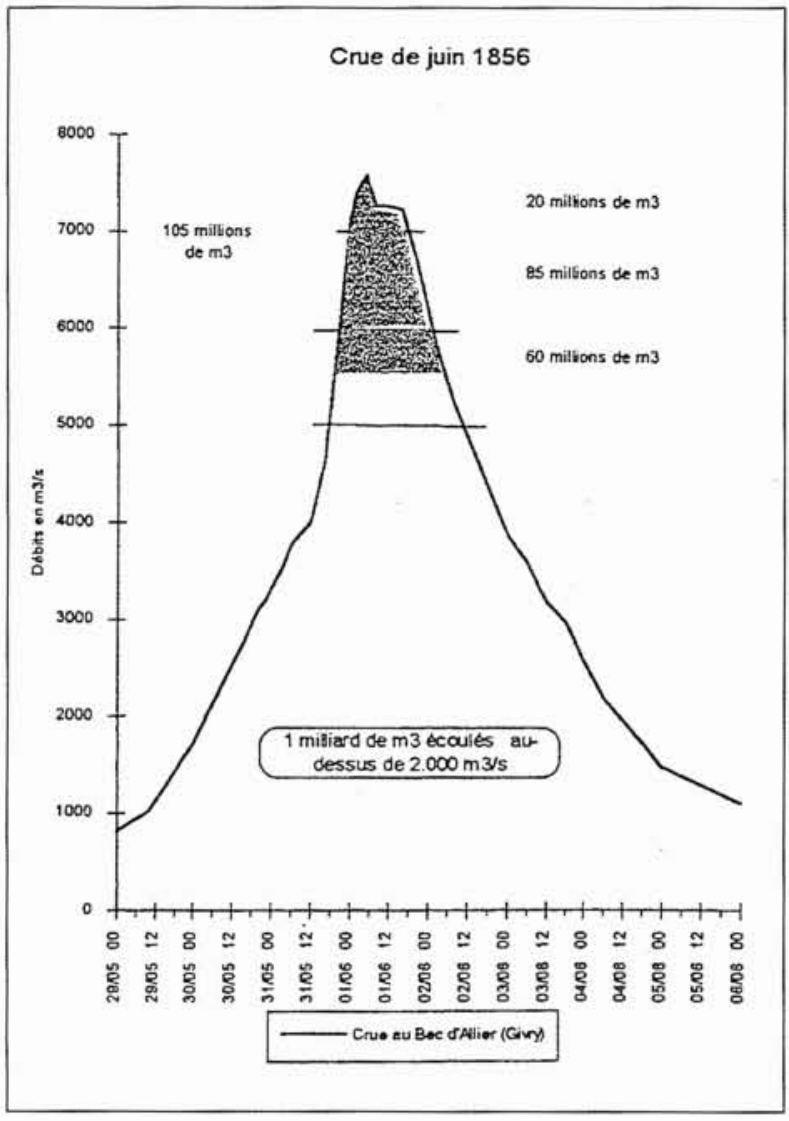

\section{Hydrogramme et volume de la crue de $\mathbf{1 8 5 6}$}

le fonctionnement des déversoirs en particulier ceux d'Ouzouer, de Jargeau et de Mazan (Meung/Loire). Ce dernier ne fonctionne plus pour une crue de type 1856, la ligne d'eau s'abaissant de plus de $2 \mathrm{~m}$ à son droit. Les crues arrivent en aval moins écrêtées et plus dangereuses (fig. 6).

Un enfoncement du lit peut, au droit de levées, nuire à leur stabilité et attaquer leur fondation. Il provoque généralement une végétalisation du lit endigué moins sollicité par les crues, nécessitant un entretien plus régulier et soigné pour maintenir une diversité écologique et assurer un moindre impact sur les niveaux d'eau en crue (cf. ci-dessus et fig. 4).

Reconquérir le lit endigué : une politique de restauration et d'entretien du lit

Tout au long du lit endigué, on constate un amortissement des crues exceptionnelles, le fleuve ne recevant aucun affluent notable sur les $300 \mathrm{~km}$ séparant le Bec d'Allier de Tours. La modélisation de 1982 montre que, sans aucune inondation des vals, le débit maximum diminue tout de même de $600 \mathrm{~m}^{3} / \mathrm{s}$ sur les $170 \mathrm{~km}$ séparant le Bec d'Allier d'Orléans et de $200 \mathrm{~m}^{3} / \mathrm{s}$ supplémentaires jusqu'à Tours.

Le niveau d'entretien influence profondément cette capacité d'écrêtement et peut induire localement des modifications de la ligne d'eau comparables à celles que génère le fonctionnement des ouvrages écrêteurs ou déversoirs. La crue de 1866, de même maximum que celle de 1856, n'a pas engendré les mêmes dégâts, en particulier à cause d'un meilleur entretien de la végétation dans les traversées de Tours et Orléans que complétait l'arrachage des oseraies encombrant le lit endigué (travaux de Z. Gasowski).

Une étude plus récente, supervisée par Z. Gasowski, montre que dans la seule traversée d'Orléans (où la Loire dispose de 


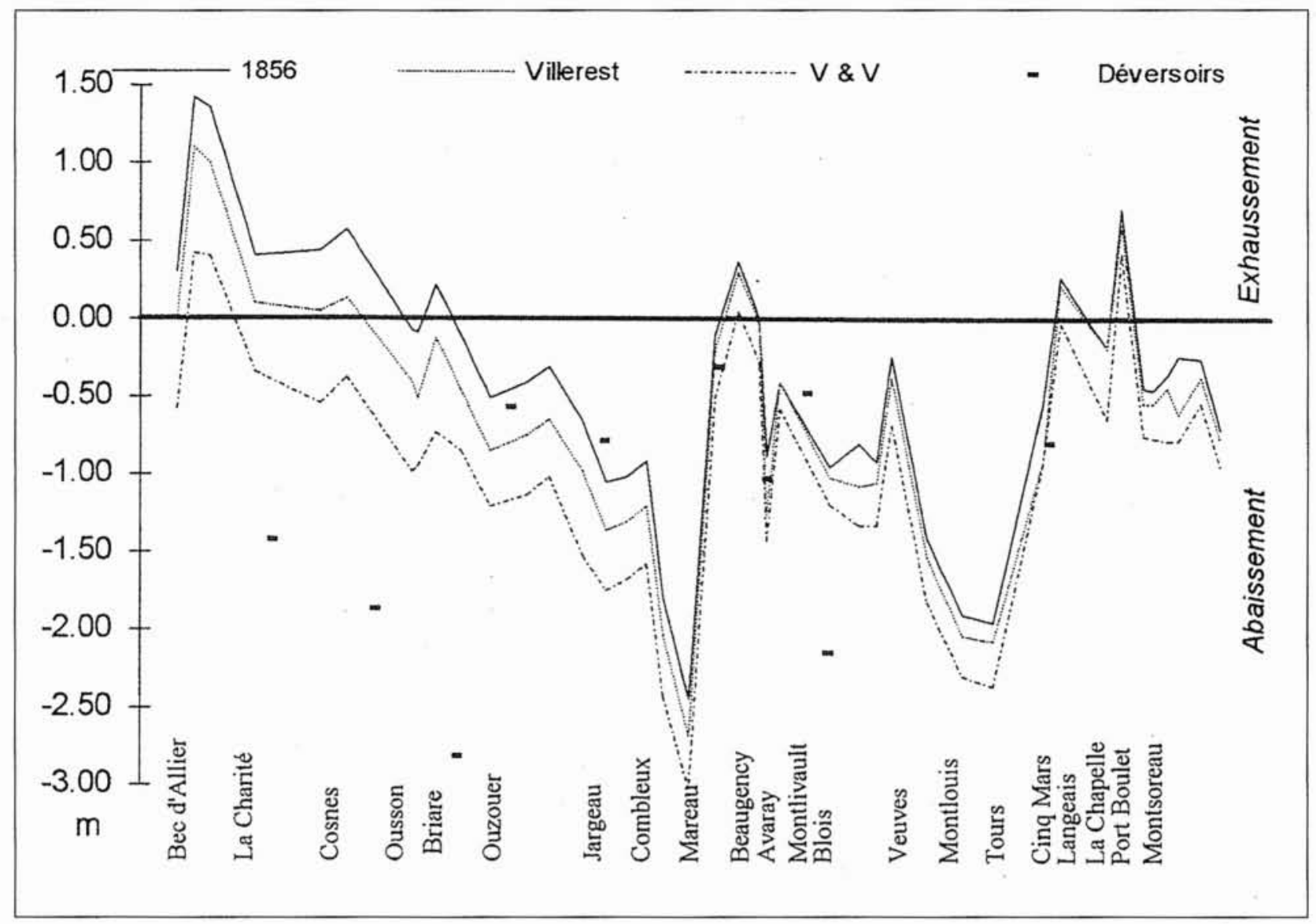

6. Différence de cote entre la ligne d'eau de la crue de 1856 s'écoulant dans une topographie relevée entre 1965 et 1980 et la ligne d'eau relevée lors de la crue de 1856

1856 : crue de 1856 sans intervention d'ouvrage écrêteur Villerest : crue de 1856, ecrêtée par le barrage de Villerest (existant)

V et V : crue de 1856, écrêtée par Villerest et le Veurdre Déversoir : cote des déversoirs existants.

moins de $400 \mathrm{~m}$ de largeur au pont George V), l'effet espéré de Villerest (estimé à $30 \mathrm{~cm}$ d'abaissement) peut se trouver annulé si la rugosité du lit y augmente de 15 à $20 \%$. L'encombrement du lit dans les sites très étroits de la Loire (La Charité, Ousson, Ouzouer, Sully, Jargeau, Orléans, Menars, Blois, Amboise, Montlouis, Tours, Ingrande, Chouzé, Saumur) peut ainsi y induire, en crue, des élévations de la ligne d'eau suffisantes pour mettre en péril les levées.

La modélisation de 1982 illustre l'impact d'une politique d'entretien au niveau local, montrant qu'une végétalisation importante de la Loire sur une longueur de $10 \mathrm{~km}$ entre le Bec d'Allier et la Charité crée une zone d'accumulation et d'écrêtement par défaut d'entretien, avec surcote locale de la crue de $1,20 \mathrm{~m}$.

\subsection{Gérer au mieux un ou des ouvrages écrêteurs de crue}

Le barrage de Villerest, ouvrage multifonctions en amont immédiat de Roanne, est le seul écrêteur à ce jour. Il abaisserait la ligne d'eau de $30 \mathrm{~cm}$ à Châteauneuf et Orléans et de $15 \mathrm{~cm}$ à Tours. Son règlement de fonctionnement prévoit de surbaisser le niveau de la retenue deux fois par an du $1^{\mathrm{er}}$ au 15 juin et du 15 septembre au $1^{\text {er }}$ décembre, période de prédilection des crues exceptionnelles.
Une étude EPALA/Service de bassin de 1985 a étudié les 80 sites recensés par Comoy pour écrêter les crues sur l'Allier. Elle a retenu le site du Veurdre, juste avant la confluence avec la Loire. Cette situation permet de voir se former la crue de l'Allier et grossir celle de la Loire, après son écrêtement par Villerest et ainsi de bien voir venir la crue avant de mettre l'ouvrage à contribution. On le fermerait uniquement pour les crues fortes et exceptionnelles et il retiendrait jusqu'à 120 millions de $\mathrm{m}^{3}$.

Ces deux ouvrages écrêteurs gérés ensemble abaisseraient la ligne d'eau en crue de $90 \mathrm{~cm}$ à Cosne, $70 \mathrm{~cm}$ à Orléans, 40 à Tours, 30 à Montsoreau et 25 aux Ponts de Cé, à condition que certains déversoirs continuent à écrêter localement. $\mathrm{Si}$ I'Etat autorise finalement la construction d'un $2^{\mathrm{c}}$ ouvrage écrêteur, l'efficacité de la gestion commune des ouvrages reposera sur un règlement très élaboré et adapté aux différentes situations de confluence de crues que l'on pourra rencontrer.

\subsection{Recourir le plus tard possible aux champs d'expansion dans les vals}

La modélisation de 1982 montre bien le rôle incontournable des vals dans l'écrêtement des crues exceptionnelles. Le tableau de la figure 7 compare trois conditions de propaga- 


\begin{tabular}{|l|c|c|c|c|c|c|c|c|}
\hline \multicolumn{2}{|c|}{} & \multicolumn{2}{|c|}{ Crue de 1856 } & \multicolumn{2}{|c|}{$\begin{array}{c}\text { Ecrêtée par } \\
\text { Villerest }\end{array}$} & \multicolumn{2}{|c|}{$\begin{array}{c}\text { Ecrêté par } \\
\text { Villerest } \\
\text { et le Veurdre }\end{array}$} & \multicolumn{2}{|c|}{$\begin{array}{c}\text { Ecrêtée par } \\
\text { Villerest } \\
\text { et le Veurdre } \\
\text { Sans vals }\end{array}$} \\
\cline { 2 - 9 } & $\mathrm{Q}_{\max }$ & $\mathrm{H}_{\max }$ & $\mathrm{Q}_{\max }$ & $\mathrm{H}_{\max }$ & $\mathrm{Q}_{\max }$ & $\mathrm{H}_{\max }$ & $\mathrm{Q}_{\max }$ & $\mathrm{H}_{\max }$ \\
\hline Le Bec d'Allier & 7600 & & 6910 & & 5750 & & 5750 & \\
Cosne & 6910 & 6,65 & 6140 & 6,22 & 5320 & 5,72 & 5490 & 6,21 \\
Chateauneuf & 6360 & 6,51 & 5800 & 6,21 & 5180 & 5,82 & 5440 & 5,97 \\
Orléans & 6230 & 5,65 & 5780 & 5,37 & 5170 & 4,97 & 5430 & 5,18 \\
Amont Blois & 5710 & & 5500 & & 5100 & & 5400 & \\
Tours & 5520 & 6,00 & 5370 & 5,87 & 4990 & 5,58 & 5390 & 5,89 \\
Montsoreau & 6250 & 6,78 & 6110 & 6,68 & 5840 & 6,48 & 6200 & 6,72 \\
Les Ponts de Cé & 6260 & 6,28 & 6120 & 6,19 & 5860 & 6,05 & 6200 & 6,10 \\
Aval Maine & 6650 & & 6510 & & 6300 & & 6300 & \\
\hline
\end{tabular}

7. Comparaison des conditions de propagation de la crue de juin 1856 avec ou sans intervention des vals et des ouvrages écrêteurs dans la topographie de la Loire relevée entre 1965 et 1980.

tion de la crue de 1856 et montre qu'à partir de Tours aucun bénéfice n'est tiré de la construction du Veurdre si on empêche simultanément toute inondation de val. Une autre simulation illustre combien il est vain de croire pouvoir s'affranchir de toute inondation, en révélant que si aucun val n'avait été inondé en 1856, la crue serait passée avec $50 \mathrm{~cm}$ de hauteur d'eau en plus à Orléans et 1 mètre de plus à Tours !

Des champs d'expansion dans les vals sont donc incontournables pour les crues exceptionnelles, mais il s'agit de solliciter uniquement ceux qui s'avéreront indispensables pour apporter une diminution sensible de la crue, de les solliciter en dernier recours et au moment le plus efficace hydrauliquement. La stratégie doit donc mettre en lumière des seuils de sollicitation des vals et définir leurs conditions optimales d'écrêtement.

Cette intervention des champs d'expansion protégera les espaces situés en aval sur le cours. La gestion des crues ne s'affranchira pas d'une solidarité hydraulique de l'amont au profit de l'aval révélée et connue ancestralement par les ligériens.

\subsection{Etablir une stratégie de gestion des crues qui orientera les stratégies de protection}

La stratégie à définir indiquera quelles combinaisons se révéleront les plus efficaces et les plus robustes pour minimiser les dégâts générés par différents niveaux de crue compris entre $4500 \mathrm{~m}^{3} / \mathrm{s}$ et $8500 \mathrm{~m}^{3} / \mathrm{s}$. Elle permettra notamment à l'Etat de prendre sa décision vis-à-vis de l'ouvrage écrêteur du Veurdre, au plus tard le 31 décembre 1998.

De cette stratégie découlera une orientation dans le renforcement des levées, pour assurer une protection optimale en fonction des zones sensibles ainsi que des vals devant conserver leur volume disponible pour un éventuel écrêtement (le volume, envahi prématurément si la levée venait à rompre avant le maximum de la crue, ne pourrait plus être éventuellement utilisé au moment du passage de la pointe la plus dommageable).
La stratégie retenue orientera aussi la politique d'entretien du lit, en précisant les niveaux d'entretien à atteindre dans les traversées d'agglomération, en localisant les zones les plus sensibles et celles où l'on peut au contraire espérer un certain écrêtement par la végétation, sans danger pour les levées. Cette politique devra améliorer le milieu naturel fluvial dont la qualité écologique s'est dégradée depuis quelques décennies. Elle profitera, en ce sens, des expériences en cours sur les "chantiers expérimentaux" d'entretien du lit supervisés par des groupes de travail locaux regroupant services de l'Etat, élus locaux, associations de protection de la nature, de pêcheurs et experts scientifiques. Elle s'articulera fortement avec le programme de restauration des milieux naturels, autre volet du plan Loire mené conjointement par les partenaires, et avec les opérations LIFE - Loire Nature.

Les travaux de restauration du lit se conduiront aussi en fonction des conclusions de la modélisation vis-à-vis de leur impact sur les crues exceptionnelles. La stratégie pourra définir une orientation et des priorités dans les travaux restant à mener.

Le système d'alerte et de secours disposera de données actualisées pour fonder sa politique.

\section{UNE DÉMARCHE ORIGINALE ET ÉQUILIBRÉE}

\subsection{Prévention, prévision, protection}

La stratégie à définir s'inscrit dans la dynamique du plan Loire, décidé le 4 janvier 1994. Elle concilie, vis-à-vis des crues exceptionnelles, la prévention, la prévision et la protection.

La prévention repose sur la publication des atlas des zones inondables, sur la définition des projets de protection qualifiés de projet d'intérêt général (PIG) et sur les modifications des plans d'occupation des sols, qu'ils impliquent. La Loire moyenne fait figure de pilote en la matière puisque tous les 
atlas y sont publiés et que les principales agglomérations ont déjà fait l'objet d'un PIG. L'Etat prend ses responsabilités et incite les collectivités à développer à nouveau la culture du risque. Il s'agit d'apprendre à vivre avec les crues mêmes les plus exceptionnelles, d'inventer un mode de développement compatible avec tous les niveaux de crues.

La prévision fait appel au réseau Cristal, outil que partagent l'Etat et l'EPALA, pour gérer les ouvrages de soutien d'étiage (Villerest, Naussac) et d'écrêtement de crues (Villerest). Il s'agit d'un réseau de collectes de données (pluie, températures, pression, débit, hauteur d'eau) sur l'ensemble du bassin versant, pour prévoir les débits de crues et d'étiage et gérer en conséquence les ouvrages. Ce réseau a montré son intérêt et son efficacité depuis dix ans qu'il existe. Il a prouvé qu'une gestion commune d'un tel outil de prévision était possible. Le plan Loire prévoit son renouvellement et une extension de son domaine géographique d'action. La stratégie de gestion à définir pourra mettre à disposition des partenaires, une modélisation simulant la propagation des crues sur la Loire moyenne. Elle concerne aussi la réactualisation des plans d'évacuation des vals inondables élaborés par les préfectures et la redéfinition des conditions d'alerte des crues par les services concernés.

La protection repose sur les politiques définies pour l'entretien du lit et le renforcement des levées. Une protection des paysages, des milieux naturels sensibles et des ouvrages patrimoniaux s'impose conjointement à ces démarches, dans l'esprit du plan Loire intervenant déjà sur ces thèmes (3 ${ }^{\text {ème }}$ chapitre du plan).

\subsection{Elaborer une stratégie hydraulique efficace et robuste}

L'agence de l'eau, l'EPALA et l'Etat, afin de définir ensemble la stratégie hydraulique, ont constitué une équipe pluridisciplinaire pour une durée limitée ; ils s'appuieront sur une modélisation hydraulique de la Loire moyenne.

L'équipe regroupe 6 personnes, pour conduire une série d'études et mener à bien le plan Loire sur deux points essentiels : la stratégie de gestion des crues et la restauration des milieux naturels. Les trois partenaires en définissent et pilotent conjointement les activités. Pour permettre aux pouvoirs politiques de définir la stratégie attendue, l'équipe s'intéressera à la fois aux conditions hydrauliques de la propagation des crues ainsi qu'aux dommages et aux impacts qu'elles génèrent.

Une modélisation hydraulique définira dans un premier temps les conditions actuelles de la propagation et celles que l'on peut attendre d'un programme adéquat de restauration et d'entretien du lit. Les trois partenaires établiront ainsi l'impact sur les crues des différents paramètres influençant sensiblement les écoulements : évolution de la topographie après les extractions des matériaux, entretien du lit, sollicitation des levées et risque de rupture, fonctionnement des déversoirs.

Elle comparera ensuite des scénarios de minimisation des impacts des crues, faisant intervenir, seules ou combinées entre elles, les solutions possibles d'écrêtement :

- mobilisation optimale du lit endigué,

- entretien du lit,

- ouvrage(s) écrêteur(s),

- mobilisation exceptionnelle de champs d'expansion dans les vals inondables, par fonctionnement de déversoir existant ou à créer.

Les trois partenaires en dégageront une ou plusieurs stratégies hydrauliques en fonction des niveaux de crue. Ils arrêteront une stratégie globale de gestion des crues en tenant compte des aspects autres qu'hydrauliques, en particulier le souci de minimiser les dommages.

\section{ㄱ. 3.3 Minimiser les dommages}

En parallèle à la démarche de modélisation hydraulique, les trois partenaires caractériseront les dommages de crues le long de la Loire moyenne et compareront leur diminution suivant les différentes stratégies jugées hydrauliquement pertinentes.

Une première étape, originale par son ampleur, consistera à bien décrire l'ensemble des dommages générés par les crues fortes et exceptionnelles, qu'il s'agisse des atteintes aux biens des ménages (habitations et mobilier), des atteintes aux tissus industriel, artisanal ou commercial, aux infrastructures de transport ou aux réseaux de distribution (eau, électricité, gaz, téléphone, ...) ou encore des impacts sur le milieu naturel et le patrimoine culturel.

Certains aspects comme l'habitat disposent d'une méthode d'évaluation. D'autres nécessiteront une démarche très prospective et novatrice, comme la caractérisation des dommages à attendre des remontées de nappe ou des dysfonctionnements de réseaux d'assainissement, de même que l'impact de telles crues sur le tissu artisanal, les équipements publics et collectifs, le milieu naturel ou la patrimoine paysager ou culturel. D'autres aspects resteront difficiles d'accès, comme l'impact sur l'économie régionale ou nationale, sachant qu'une inondation de type 1856 interrompra pratiquement toute liaison routière ou ferroviaire à travers la Loire pendant 2 à 3 jours (ou plus), les accès à tous les viaducs et ponts se trouvant noyés, et qu'elle induira un grand nombre de cessations partielles d'activités même dans des zones non inondées (impossibilité d'accès par le personnel, rupture de fourniture, plan Orsec).

L'équipe pluridisciplinaire conduit tout ce travail de caractérisation, en constituant une base de données des enjeux. La base décrit l'occupation des sols et recense l'ensemble des implantations humaines, des activités économiques et des équipements publics. Elle décrit plus finement, avec l'aide des collectivités concernées, les impacts dans les vals de Tours et Orléans. Ce recensement servira à cibler les moyens de réduire la vulnérabilité en même temps que l'aléa hydraulique. La stratégie pourra ainsi déboucher sur une politique de diminution des enjeux exposés.

La stratégie engagera une politique sur plus de 20 à 30 ans ; la décision finale devra s'inscrire dans la durée et prendre en compte l'évolution à moyen terme de l'enjeu exposé et les générations futures. Les trois partenaires devront trouver les outils de comparaison des scénarios possibles, qui illustrent l'impact des différentes possibilités sur le moyen terme.

\subsection{Informer et débattre pour faire naître un consen- sus}

La décision finale reviendra aux autorités, dont le choix sera d'autant plus serein et avisé qu'elles seront complètement et correctement informées des tenants et aboutissants de leur option. Elaborer une communication fiable, correcte et facilement compréhensible sur un sujet aussi complexe est aussi un défi que relèvent les trois partenaires. Ils doivent se donner les moyens d'arriver à cette fin.

La stratégie retenue se mettra en place dans le cadre d'une concertation qui vise à aboutir à un consensus ligérien. Celui-ci traduira notamment, dans la stratégie retenue, la solidarité amont-aval sur laquelle reposera tout écrêtement efficace des crues. 